\title{
Schottky effect at a metal-polymer interface
}

\author{
G. L. J. A. Rikken, D. Braun, E. G. J. Staring, and R. Demandt \\ Philips Research Laboratories, Prof. Holstlaan 4, NL-5656 AA Eindhoven, The Netherlands
}

We report the observation of the Schottky effect at the interface between a metal and a semiconducting polymer by means of internal photoemission spectroscopy. The bias dependence of the barrier provides information on the electrical properties of the polymer.

Organic thin films find more and more applications in (opto)electronic devices, but still very little is known about the electronic properties of their interfaces. The interface properties between metals and organic (semi)conductors are especially, although the utmost technological importance, not well understood. One reason is the very dissimilar character of these materials' classes and the formalisms used to describe them. Furthermore, there are few interface specific characterization techniques. This makes it difficult to prepare well defined and reproducible interfaces and as a result, so far no extensive experimental basis for theoretical modeling has been established. This letter reports the observation of image force potential lowering of the potential batrier (the so-called Schottky effect) at the interface between a metal and a semiconducting polymer by means of internal photoemission spectroscopy. From this effect, information on the electrical properties of the polymer can be obtained.

The energy-band diagram of an ideal metalsemiconductor-metal heterostructure is schematically shown in Fig. 1, with the inset illustrating the Schottky effect, ${ }^{1}$ under the assumption that the free-carrier concentration in the semiconductor is so low that the resulting screening length exceeds the semiconductor thickness $d$. If the metal is negatively biased, the image force potential $\phi_{\text {im }}$ causes a lowering of the barrier height for electron injection from the metal into the semiconductor by $\Delta \phi$ and the turning point of the injection barrier is located at a distance $x_{m}$ from the interface. It can be shown that in the given units ${ }^{1}$

$$
\Delta \phi=\sqrt{\frac{q F}{4 \pi \epsilon_{s} \epsilon_{0}}}[V] \quad x_{m}=\sqrt{\frac{q}{16 \pi \epsilon_{s} \epsilon_{0} F}}[m],
$$

where $\epsilon_{s}$ is the dielectric constant of the semiconductor, $q$ the electron charge, and $F$ the electric field in the semiconductor, resulting from space-charge effects and externally applied bias. For a semiconductor with uniform ionized impurity density $N$, the potential lowering becomes

$$
\Delta \phi=\left[\frac{q^{3} N\left(V-V_{b i}+k T / q\right)}{8 \pi^{2} \epsilon_{s}^{3} \epsilon_{0}^{3}}\right]^{1 / 4} .
$$

This simple physical picture has been proven correct by internal photoemission spectroscopy (IPE) measurements on Au-Si junctions. ${ }^{2}$ Since then, the model has been refined ${ }^{3-5}$ to include band bending, tunneling, phonon scattering, and inhomogeneities, but the essence remains that there is a potential barrier of which position and height can be adjusted by an external electric field.

Photoemission (PE) is by now a well established technique to study interfaces of inorganic heterojunctions. ${ }^{6.7}$ When using x-ray (XPS), deep UV (UPS), or synchrotron light sources, the kinetic-energy dependence of the escape depth of the photoelectrons allows for depth resolution on a nanometer length scale. The energy resolution in that case is limited to typically $50-100 \mathrm{meV}$ and only interfaces that are very close to the surface can be studied. Such techniques have also been applied to metal-polymer interfaces. One finds chemical binding of the metal atoms to the polymer or impurities $^{8-10}$ or doping of the polymer. ${ }^{11,12}$ The corresponding electronic band structure has remained undetermined. With visible light, $\mathrm{PE}$ at higher energy resolution can be performed, but with no depth resolution. This has also been applied to metal-polymer junctions ${ }^{13,14}$ in order to determine the polymer band structure and band offsets. It turns out that the offsets between the bands in the polymer and the Fermi level of the metal are reasonably well described by the electron affinity rule, i.e., continuity of the vacuum level across the interface. These findings are in strong contrast to the case of metal-inorganic-semiconductor interfaces, where the

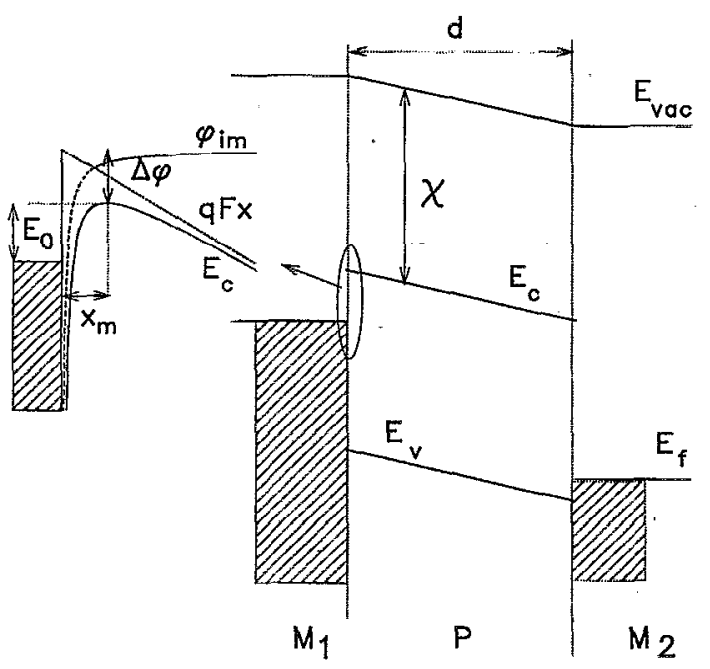

FIG. 1. Schematic view of band structure of a metal-polymer-metal heterostructure, with parameters approximatcly corrcct for the casc Ca-OPPV-ITO, Ca negatively biased. Inset shows the band structure- near a metal-polymer interface, illustrating the Schottky effect. 


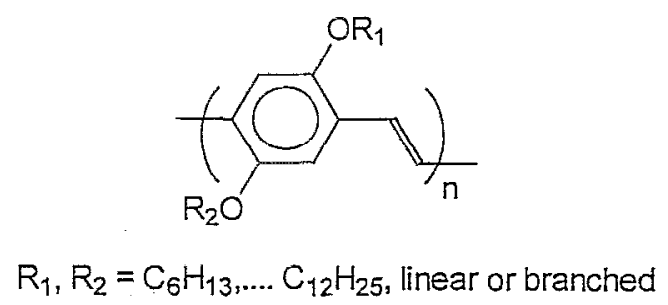

FIG. 2. Chemical structure of the semiconducting polymer OPPV used in this experiment.

Schottky barriers in general do not obey the electron affinity rule (see, e.g., Ref. 1) and it is unclear how they relate to the XPS and UPS observations of the formation of interface layers at metal-polymer interfaces. Combining the external electric-field dependence of the Schottky effect with highresolution visible-light IPE provides a method to probe electronic properties near a metal-semiconductor interface, as shown below.

The semiconducting polymer used in this experiment, shown in Fig. 2, is a soluble, di-alkoxy substituted poly (paraphenylene-vinylene) (OPPV), a member of a wellknown class of semiconducting polymers ${ }^{15}$ that was synthesized according to the method described by Swatos et al. ${ }^{16}$ and not intentionally doped. Samples are prepared on glass substrates covered with patterned indium-tin oxide (ITO) electrodes. ${ }^{17}$ These materials are transparent to the wavelengths used in this experiment and therefore do not contribute to the photocurrent. Polymer films are spin-coated and provided with 250-nm-thick calcium contacts by vapor deposition at a rate of $1 \mathrm{~nm} / \mathrm{s}$, with the substrate at room temperature. All these processing steps are carried out in a nitrogen atmosphere with oxygen and water vapor concentrations below $1 \mathrm{ppm}$. Finally, the samples are sealed with aluminum and an epoxy resin, stored and transported in an inert atmosphere, and measured in a vacuum chamber (pressure $\leqslant 10^{-6}$ Torr) at room temperature. IPE spectroscopy is performed by phase-sensitive detection of the photocurrent response to a chopped optical beam of tunable photon energy $E_{\mathrm{ph}}$, smaller than the polymer band gap. This technique ensures that only current due to electron or hole photoemission out of the $\mathrm{Ca}$ into the polymer is detected. Figure 3 shows a typical result, together with a schematic setup.

Standard photoemission theory ${ }^{18}$ predicts that the $\mathrm{PE}$ quantum yield (QY) should be proportional to $\left(E_{\mathrm{ph}}-E_{0}\right)^{2}$, where $E_{0}$, as defined in Fig. 1, is the barrier height between the Ca electrode Fermi level and the maximum of the polymer conduction band and $E_{\mathrm{ph}}$ is the photon encrgy, for the case that the $\mathrm{Ca}$ is negatively biased. This relation is very clearly observed in Fig. 3. By extrapolating to zero QY, one can obtain $E_{0}$ as a function of the externally applied bias $V$. The internal electric field $F$ in the polymer results from this applied voltage plus a built-in voltage $V_{\mathrm{bi}}$ that equals the work-function difference of the two metallic electrodes. $V_{\mathrm{bi}}$ can be determined by measuring the open circuit photovoltage under intense illumination with photon energies above the polymer band gap $(2 \mathrm{eV}$ ) or alternatively, by determining the bias voltage at which the photocurrent for above band-

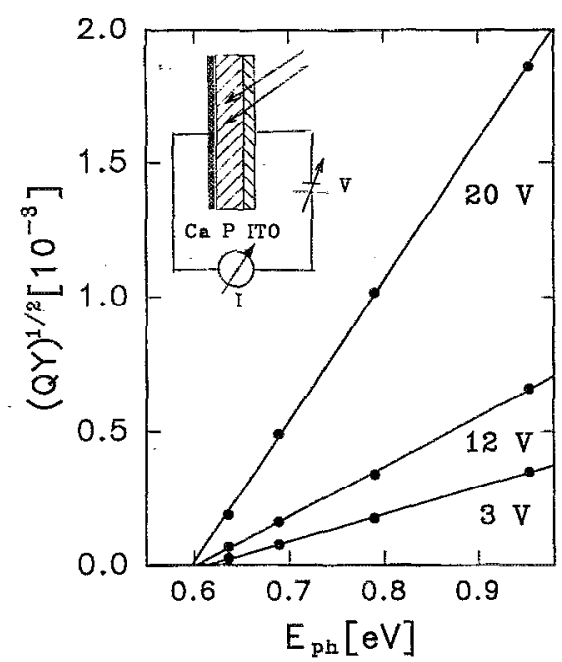

FIG. 3. Typical result for IPE measurement of quantum yield vs photon energy on a Ca-OPPV-ITO multilayer of $0.73 \mu \mathrm{m}$ polymer thickness at several applied voltages with the $\mathrm{Ca}$ electrode negatively biased with respect to the ITO. Lines are least-square fits and their extrapolation yields $E_{0}$. Inset schematically shows the experimental setup.

gap illumination is minimal (flatband condition). Both measurements yield a value of $1.4 \mathrm{~V}$ with the ITO positive with respect to the $\mathrm{Ca}$, with a typical spread between samples of $0.1 \mathrm{~V}$. This value agrees well with the difference in the workfunctions of Ca (Ref. 19) (2.89 eV) and ITO (Ref. 20) (4.4 eV). Figure 4 shows the observed barrier height $E_{0}$ as a function of $\left(V-V_{\mathrm{bi}}\right)^{1 / 2}$. The zero-field barrier of $0.615 \mathrm{eV}$ is the difference between the Ca Fermi level and the OPPV conduction band far away from the interface. Variations of $0.05 \mathrm{eV}$ were observed between the different samples. The electron affinity $\chi$ (see Fig. 1) of a similar polymer is reported to be $2.7 \mathrm{eV}^{21}$ The electron affinity rule would then predict a barrier of $0.2 \mathrm{eV}$. Vice versa, if the electron affinity rule applies here, we deduce an electron affinity of $2.28 \mathrm{eV}$

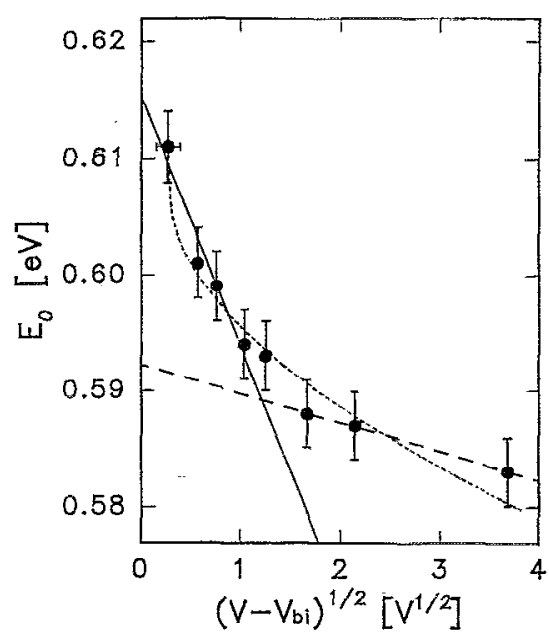

FIG. 4. Barrier height $E_{0}$, as deduced from IPE measurements, as a function of $\left(V-V_{\mathrm{bj}}\right)^{1 / 2}$ for a multilayer as in Fig. 3. Solid line is a least-squares fit to the data at the lowest four voltages; long dashed line is a least-squares fit to the data at the highest three voltages. Short dashed line is a fit to Eq. (2). 
for our polymer. As the electron affinity may strongly depend on the exact polymer properties, it is at this time not possible to draw conclusions on the validity of the electron affinity rule for this case. In addition, the presence of interface states may change the interpretation. Clearly, more data on different well defined metal-polymer combinations are required to establish a database on which models can be founded.

For the observed bias dependence of $E_{0}$, two possible explanations can be put forward. If the polymer is only intrinsically conducting (undoped), then $F=\left(V-V_{\mathrm{bi}}\right) / \epsilon_{s} d$ and Eq. (1) predicts a linear relation between $\Delta \phi$. and $F^{1 / 2}$. This is indeed observed in Fig. 4 for low applied bias. This corresponds to a situation where the barrier maximum is located far away from the interface. Using $\epsilon_{s}=3.0$, as determined from capacitance measurements, we deduce from Eq. (1) a slope of $15 \mathrm{meV} / \mathrm{V}^{1 / 2}$. The observed low bias slope is $20 \pm 3$ $\mathrm{meV} / \mathrm{V}^{1 / 2}$, which is in fair agreement. Although there are other possible causes for the field dependence of the barrier height, ${ }^{22}$ none of those has a linear relation between $\Delta \phi$ and $F^{1 / 2}$. As the bias is increased, the behavior turns over into a much weaker dependence (the long dashed line in Fig. 4 has a slope of $3 \pm 3 \mathrm{meV} / \mathrm{V}^{1 / 2}$ ). Therefore, at high bias, when the barrier maximum is located close to the interface, increasing the bias voltage results in a much smaller electric-field increase at the barrier maximum (and therefore a smaller barricr lowering) than at low bias, when the barrier maximum is far away from the interface. At the lowest applied bias in Fig. 4 , the barrier maximum, according to Eq. (1), is located 30 $\mathrm{nm}$ from the interface, whereas as at $\left(V-V_{\mathrm{bi}}\right)^{1 / 2}=1.2 V^{1 / 2}$, where the bias dependence turns over, it is located $6.5 \mathrm{~nm}$ from the interface. This means that at least between 30 and $6.5 \mathrm{~nm}$ from the interface, the band structure is reasonably well described by Eq. (1) and the assumption underlying it; i.e., that the Ca-OPPV junction behaves as an ideal metalundoped semiconductor junction, whereas in a layer of 6.5 $\mathrm{nm}$ thickness, adjacent to the $\mathrm{Ca}$ electrode, this description is no longer valid. One might expect the presence of (partly filled) interface states to be responsible for this, but one can show ${ }^{1,22}$ that such interface charge leads to an additional band offset that is essentially independent of electric field. Interface states may therefore be invoked to explain deviations from the electron affinity rule, but not the turn over in Fig. 4. One could qualitatively explain the result by assuming that in a layer of $6.5 \mathrm{~nm}$ thickness adjacent to the $\mathrm{Ca}$ electrode, the dielectric constant of the polymer is very large, $\epsilon_{s} \geqslant 15$, or the conductivity is much larger than in the bulk of the polymer, by at least a factor of 25 . As there is no reason to expect the dielectric constant to change this much, one would then have to conclude that the conductivity in the polymer near the $\mathrm{Ca}$ interface is larger than in the bulk, most likely because of doping with $\mathrm{Ca}$ atoms in agreement with the findings of Salaneck et al. ${ }^{12}$ Strictly speaking, Eq. (1) does not apply to such a situation and one should derive new expressions for $x_{m}$ and $\Delta \phi$ based on the modified potential profile. At this stage, the accuracy of the measurements does not warrant such a procedure.

Alternatively, one might expect some unintentional doping and therefore, a bias dependence of the barrier height that is described by Eq. (2). The best fit of this relation to our experimental data is also shown in Fig. 4 (short dashed line) and agreement is reasonable for an ionized impurity concentration of $2.10^{13} \mathrm{~cm}^{-3}$. This number is of the same order of magnitude as reported for a similar polymer. ${ }^{23}$ Our measurements of the bias dependence of the Schottky barrier can therefore be quantitatively explained by either an undoped polymer, with a doped interface layer, or a homogeneous, slightly doped polymer. More, and more accurate, measurements are required to discern between the two explanations.

In summary, we have reported for the first time the observation of the Schottky effect at a metal-semiconducting polymer interface by means of IPE. The zero-field barrier height at the Ca-OPPV interface was $0.615 \mathrm{eV}$, but independent data are needed to judge the applicability of the electron affinity rule for band alignment. From the bias dependence of the barrier height, we infer either that the polymer is undoped, with a doped layer near $(6.5 \mathrm{~nm})$ the $\mathrm{Ca}$ interface, or that a homogeneous ionized impurity concentration of $2.10^{13}$ $\mathrm{cm}^{-3}$ is present in the polymer. Clearly, IPE is a valuable technique to study metal-polymer interfaces, and further measurements are in progress to address the barrier height between different metal-polymer combinations, and its bias dependence.

We gratefully acknowledgc H. Wynberg and W. ten Hoeve (Syncom BV) for preparation of the monomers, Y. Kessener for technical assistance, and D. de Leeuw, E. J. Lous, and N. Pulsford for critical reading of the manuscript. Part of this work was financed by the EEC under BRITE-EURAM BRE2-CT93-0592.

${ }^{1}$ S. M. Sze, Physics of Semiconductor Devices (Wiley, New York, 1981).

${ }^{2}$ S. M. Sze, C. R. Crowell, and D. Kahng, J. Appl. Phys. 35, 2534 (1964).

${ }^{3}$ C. Kao, C. L. Anderson, and C. R. Crowell, Surf. Sci. 95, 321 (1980).

${ }^{4}$ C. Wu, J. Appl. Phys. 52, 2909 (1981).

${ }^{5}$ R. T. Tung, Phys. Rev. B 45, 13509 (1992).

${ }^{6}$ Heterojunctions Band Discontinuities: Physics and Device Applications, edited by F. Capasso and G. Margaritondo (North Holland, Amsterdam, 1987).

${ }^{7}$ G. Margaritondo, J. Vac. Sci. Technol. B 11, 1362 (1993).

${ }^{8}$ T. R. Nguyen, H. Ettaik, S. Lefrant, G. Leising, and F. Stelzer, Synth. Metals 38, 69 (1990).

${ }^{9}$ Y. Gao, K. T. Park, and B. R. Hsieh, J. Appl. Phys. 73, 7894 (1993).

${ }^{10}$ P. Dannetun, M. Logdlund, M. Fahlman, M. Boman, S. Stafstrom, W. R. Salaneck, R. Lazzaroni, C. Frederikson; J. L. Bredas, S. Graham, R. H. Friend, A. B. Holmes, R. Zamboni, and C. Taliani, Synth. Met. 55-57, 212 (1993).

${ }^{11}$ M. Fahlman, D. Beljonne, M. Lögdlung, R. H. Friend, A. B. Holmes, J. L. Bredas, and W. R. Salaneck, Chem. Phys. Lett. 214, 327 (1993).

${ }^{12}$ W. R. Salaneck (private communication).

${ }^{13} \mathrm{~J}$. Mort and A. Lakatos, J. Non-Cryst. Solids 4, 117 (1970).

${ }^{14} \mathrm{G}$. Giro and P. Marco, Thin Solid Films 59, 91 (1979).

${ }^{15}$ See, e.g., Proceedings of the International Conference on Synthetic Metals [Synth. Metals 55-57 (1993)], and references therein.

${ }^{16}$ W. J. Swatos and B. Gordon, Polym. Prepr. 31, 505 (1990).

${ }^{17}$ D. Braun, A. J. Heeger, and H. Kroemer, J. Electron. Mater. 20, 945 (1991).

${ }^{18}$ R. H. Fowler, Phys. Rev. 38, 45 (1931).

${ }^{19} \mathrm{CRC}$ Handbook of Chemistry and Physics, edited by D. R. Lide (CRC Boca Raton, 1991).

${ }^{20}$ D. L. Feucht, J. Vac. Sci. Technol. 14, 57. (1977)

${ }^{21}$ M. Lögdlund, W. R. Salaneck, S. Stafstrom, D. D. C. Bradley, R. H. Friend, K. E. Ziemelis, G. Froyer, D. B. Swanson, A. G. MacDiarmid, G. A. Arbuckle, R. Lazzaroni, F. Meyeres, and J. L. Bredas, Synth. Met. 43, 1315 (1991)

${ }^{22}$ E. H. Rhoderick and R. H. Williams, Metal-Semiconductor Contacts (Clarendon, Oxford, 1988).

${ }^{23}$ D. Bradley (private communication). 